\title{
Cuidados de enfermagem na prevenção de lesão por pressão em idosos internados na
}

\section{unidade de terapia intensiva}

\author{
Nursing care in the prevention of pressure injury in elderly hospitalized in the intensive care unit \\ Atención de enfermería en la prevención de lesiones por presión en ancianos hospitalizados en
}

unidad de terapia intensiva

Recebido: 23/11/2021 | Revisado: 01/12/2021 | Aceito: 03/12/2021 | Publicado: 04/12/2021

\author{
Elaine Cristina Sampaio \\ ORCID: https://orcid.org/0000-0001-5605-2860 \\ Centro Universitário FAMETRO, Brasil \\ E-mail: elaine_csampaio@hotmail.com \\ Irlane Ferreira França \\ ORCID: https://orcid.org/0000-0003-0878-6776 \\ Centro Universitário FAMETRO, Brasil \\ E-mail: irlanef84@gmail.com \\ Leandro da Cruz Silva \\ ORCID: https://orcid.org/0000-0002-1741-5398 \\ Centro Universitário FAMETRO, Brasil \\ E-mail: leandrocruzzz2016@gmail.com \\ Graciana de Sousa Lopes \\ ORCID: https://orcid.org/0000-0003-3615-9040 \\ Centro Universitário FAMETRO, Brasil \\ E-mail: gracilopess@hotmail.com
}

\begin{abstract}
Resumo
A Lesão por pressão (LPP) é o resultado da perda de integridade da pele e tecidos subjacentes que ocorre quando há aumento da pressão sob a pele, também está relacionada ao com a fricção e cisalhamento. Desse modo, as ações de prevenção dos profissionais de enfermagem visam impedir o surgimento e progressão deste agravo. Sendo assim, este estudo trata-se de uma revisão bibliográfica do tipo revisão integrativa da literatura, que buscou artigos nas bases de dados Biblioteca Científica Eletrônica Online (SCIELO), Literatura Latino Americana e do Caribe em Ciências da Saúde (LILACS) e Bases de Dados de Enfermagem (BDENF) e elaborou uma síntese acerca dos resultados encontrados. Como as principais contribuições de enfermagem para promoção da prevenção da LPP, está a aplicação da escala de Braden, mudança de decúbito, diminuição da umidade da pele, aplicação de coberturas de fibra e hidrocoloide, educação em saúde para os pacientes e atualização profissional paar os enfermeiros.
\end{abstract}

Palavras-chave: Lesão por pressão; Enfermagem; UTI; Idosos.

\begin{abstract}
Pressure Injury (LPP) is the result of the loss of integrity of the skin and underlying tissues that occurs when there is increased pressure under the skin, it is also related to friction and shear. Thus, the preventive actions of nursing professionals aim to prevent the emergence and progression of this problem. Therefore, this study is a bibliographic review of the integrative literature review type, which sought articles in the Electronic Scientific Library Online (SCIELO), Latin American and Caribbean Literature in Health Sciences (LILACS) and Databases databases. Nursing Data (BDENF) and elaborated a synthesis of the results found. The main nursing contributions to promote the prevention of PPL are the application of the Braden scale, change of position, reduction of skin moisture, application of fiber and hydrocolloid dressings, health education for patients and professional updating for the nurses.
\end{abstract}

Keywords: Pressure Injury; Nursing; UTI; Elderly.

\section{Resumen}

La lesión por presión (LPP) es el resultado de la pérdida de integridad de la piel y los tejidos subyacentes que se produce cuando hay una mayor presión debajo de la piel, también está relacionada con la fricción y el cizallamiento. Así, las acciones preventivas de los profesionales de enfermería tienen como objetivo prevenir la aparición y progresión de este problema. Por tanto, este estudio es una revisión bibliográfica del tipo revisión integradora de la literatura, que buscó artículos en las bases de datos de la Biblioteca Científica Electrónica en Línea (SCIELO), Literatura Latinoamericana y del Caribe en Ciencias de la Salud (LILACS) y Bases de Datos. Datos de Enfermería (BDENF) y elaborados una síntesis de los resultados encontrados. Los principales aportes de la enfermería para promover la prevención de la LPP son la aplicación de la escala de Braden, el cambio de posición, la reducción de la humedad de la piel, la aplicación de 
apósitos de fibra e hidrocoloides, la educación en salud para los pacientes y la actualización profesional para los enfermeros.

Palabras clave: Lesión por presión; Enfermería; UCI; Mayores.

\section{Introdução}

O envelhecimento é um processo fisiológico que ocorre durante toda a vida, seu marco estende-se desde a concepção até após a morte, tornando, com isso, é impossível identificar a partir do momento em que uma pessoa é classificada como idosa. Contudo, é necessário estabelecer limites cronológicos para estudos e planejamentos administrativos. Portanto, para a Organização Mundial da Saúde (OMS), caracteriza-se como idoso qualquer pessoa com idade igual ou superior a 65 anos, essa classificação possui exceção nos países subdesenvolvidos ou em desenvolvimento, como o Brasil, onde a faixa etária do idoso inicia-se aos sessenta anos de idade (Galvão, 2016).

O envelhecer, inevitavelmente, está associado à comorbidades e determinadas limitações, sendo elas físicas, psicológicas e sociais. Essa realidade faz com que o consumo dos serviços de saúde, consequentemente, cresça. A integralidade do cuidado ao idoso, isto é, compreender suas funções e proporcionar o cuidado adequado, permeia desafios operacionais, a escassez de recursos e/ou pessoas (Santos et al., 2016).

As patologias e limitações surgem, e é nesse contexto que os profissionais de saúde inseridos podem promover saúde e garantir que esse envelhecer seja ativo e mesmo ocorrendo forma gradual e irreversível, ocorra com a manutenção da qualidade de vida assim como é determinado a partir das políticas públicas de saúde existentes. Garantir cada vez mais expectativa de vida saudável da população idosa diminuindo sua vulnerabilidade (Mallmann et al., 2015).

Destacando o olhar sobre a família, sobre o círculo social que ele possa estar inserido, formando esse elo ao atendimento multidisciplinar, garantindo assim o direito à saúde através de uma sistematização da assistência com base em um cuidado humanizado (Medeiros et al. 2017).

A lesão por pressão (LPP) é uma lesão de pele ou tecidos moles resultantes da hipóxia celular, que pode provocar necrose nos tecidos devido à pressão ou pressão com fricção e cisalhamento, podendo apresentar-se também em pele íntegra ou ulceração aberta, dessa maneira a LPP é encontrada em localidades próximas a ossos ou regiões do corpo em que se utiliza algum de dispositivo médico ou artefato (Brasil, 2013).

A hospitalização é um evento de adaptação que ocasiona alterações na rotina do indivíduo e tende a ser angustiante devido a perda da autonomia, com isso os pacientes elevam os níveis de estresse, ansiedade e isso impacta os familiares diretamente (Feitosa, 2015). O ambiente não é acolhedor, os ruídos dos monitores e bombas de infusão prejudicam o sono, dispositivos médicos estão 24 horas acoplados no corpo, são realizados procedimentos invasivos, esses e outros fatores estressores contribuem para a não adequação a unidade hospitalar. Dessa maneira é comum que no momento de hospitalização o idoso fique com sinais de tristeza, desesperança e medo (Andrade, Marcon, Silva, 2013).

Portanto, a prevalência de LPP tem aumentado nos últimos anos devido o envelhecimento populacional, sendo necessário avanços tecnológicos que podem ser empregados na área da saúde, o que aumenta a expectativa de vida dos pacientes antes acometidos por doenças letais e que agora convivem com situação de saúde de forma crônica(Teixeira et al., 2012).

O cuidado em saúde é um direito do idoso e o mesmo deve ser realizado de forma digna e com qualidade científica. O enfermeiro ao realizar assistência em saúde buscar garantir esse direito da forma mais humanizada possível, preservando as características do indivíduo biopsicossocial. O foco do atendimento não deve estar somente em um processo curativo onde a saúde se relaciona com uma ausência patológica e sim em todos os demais fatores que podem influenciar nesse processo, frente às incapacidades e no declínio funcional que o idoso possa vir a ter (Vieira et al, 2020).

A qualidade da assistência de enfermagem, deve ser baseada em uma ciência que garanta a forma integral o atendimento eficaz, com fins resolutivos e preventivos afim de melhorar a qualidade da internação dos pacientes, além de identificar as 
necessidades de cuidado para estes idosos e os capacitando para posterior reabilitação das suas atividades (Colombrini, 2016).

A presença ou a ausência de LPP pode ser definida como um marcador de qualidade assistencial, tendo em vista que esses dados contribuem coma formação de políticas públicas, elaboração de um plano de cuidados e permite traçar uma comparação com outras instituições. Assim, a incidência da LPP varia de acordo com o ambiente clínico e as características do paciente, dessa maneira quando se trata de pacientes hospitalizados ou daqueles pacientes idosos ou não que necessitam de cuidados institucionais a longo prazo estas LPP ocorrem com maior frequência (Sanders, 2012).

Diante da temática exposta a prática e a destreza é total relevância, onde se faz necessário o embasamento teórico científico, a educação continuada juntamente com treinamento da equipe se faz necessário como forma de capacitação profissional contínua, no cuidado e prevenção dos agentes causadores de lesões por pressão na unidade hospitalar.

Portanto, o objetivo geral deste estudo foi identificar os principais cuidados de enfermagem para prevenir o surgimento de lesões por pressão em pacientes idosos internados na UTI e os objetivos específicos foram: Descrever as atribuições do enfermeiro frente ao paciente idoso com lesão por pressão e relatar as medidas de prevenção e fatores de risco para o surgimento de LPP encontradas na literatura.

\section{Metodologia}

Trata-se de um estudo descritivo, do tipo Revisão Integrativa da Literatura (RIL), que possui como objetivo reunir matéria bibliográfico acerca de uma temática e através dos resultados obtidos traçar um comparativo do que já foi publicado e responde as questões norteadoras de um estudo (Sousa et al., 2017).

Para coleta de dados, inicialmente foram selecionadas as palavras-chave que localizadas através dos Descritores em Ciências da Saúde (DeCS), estas foram: Enfermagem, Idoso, UTI e Lesão por pressão, posteriormente os DeCS em conjunto com o operador booleano AND foi posicionado na aba de busca das seguintes bases de dados: Biblioteca Científica Eletrônica Online (SCIELO), Literatura Latino Americana e do Caribe em Ciências da Saúde (LILACS) e Bases de Dados de Enfermagem (BDENF) mediantes os seguintes descritores, conforme apresentado na Figura 1.

Figura 1 - Fluxograma das etapas de seleção dos artigos para revisão.

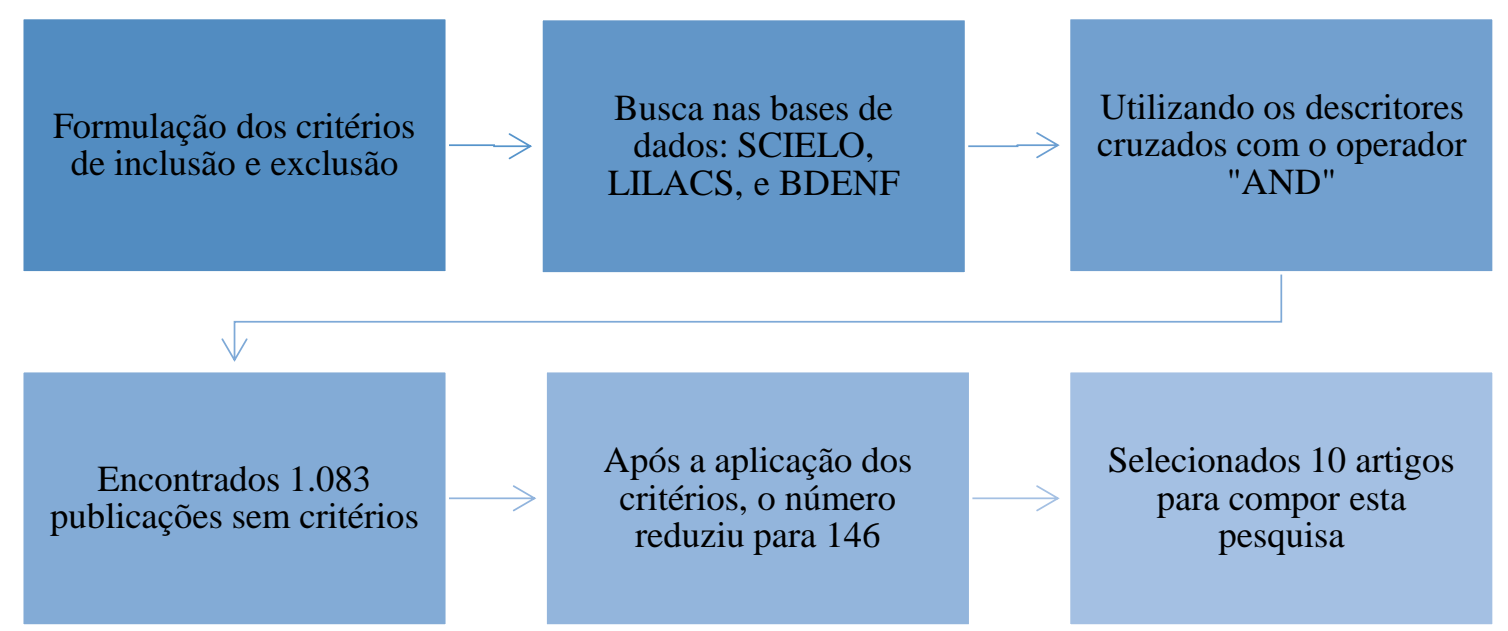

Fonte: Sampaio et al. (2021). 
Para compor os critérios de inclusão, só foram inseridos os artigos originais publicados disponíveis na íntegra, nos idiomas português e inglês, com ano de publicação entre 2015 a 2020 e que estavam relacionados ao objetivo deste estudo.

Portanto, foram descartados devido inelegibilidade os trabalhos cujo material não estava disponível ou com textos incompletos, estudos com estrutura de monografias, dissertações e teses, publicados fora do período proposto e em demais idiomas. Após a pré-seleção dos artigos obtidos, foi realizada a leitura de todo o material para descarte de duplicidade e inserção nesta revisão.

\section{Resultados}

Os artigos inicialmente encontrados nas bases de dados após a inserção das palavras-chave totalizaram 1.383, com a aplicação dos filtros e exclusão dos trabalhos duplicados, ou seja, os que apareciam duas ou mais vezes nas bases bibliográficas, restaram 10 artigos científicos originais que cumpriram as exigências e normas da revisão integrativa e que possuíam relação direta com a temática abordada. No Quadro 1 está representado as informações principais dos artigos que compuseram o presente estudo.

Quadro 1 - Tabela de Coleta de Dados.

\begin{tabular}{|c|c|c|c|c|}
\hline & TÍTULO & AUTOR/ ANO & $\begin{array}{c}\text { BASE DE } \\
\text { DADOS }\end{array}$ & RESULTADOS \\
\hline 1 & $\begin{array}{l}\text { Lesão por fricção em } \\
\text { idosos internados: estudo } \\
\text { transversal }\end{array}$ & GRDEN et al. 2020 & LILACS & $\begin{array}{l}\text { Da população de estudo, } 10,5 \% \text { apresentaram lesão por } \\
\text { pressão derivado do processo de fricção, os pesquisadores } \\
\text { também conseguiram relacionar o aparecimento de LPPs } \\
\text { com o índice de massa corpórea diminuído dos idosos. }\end{array}$ \\
\hline 2 & $\begin{array}{l}\text { Adesão da enfermagem } \\
\text { ao protocolo de lesão por } \\
\text { pressão em unidade de } \\
\text { terapia intensiva }\end{array}$ & $\begin{array}{l}\text { SNACHES et al., } \\
2018\end{array}$ & BDENF & $\begin{array}{c}\text { A equipe de enfermagem aderiu as práticas de prevenção e a } \\
\text { mais significativa foi a mudança de decúbito a cada } 2 \text { horas, } \\
\text { feita de forma correta, para controlar o tempo, a instituição } \\
\text { investiu em um relógio específico para avisar aos } \\
\text { enfermeiros a hora exata de mudar a posição do paciente. } \\
\text { Além disso a equipe trabalhou para retirar o paciente do leito } \\
\text { o mais breve possível, documentar os casos de LPP e realizar } \\
\text { capacitação profissional. }\end{array}$ \\
\hline 3 & $\begin{array}{l}\text { Custos de coberturas para } \\
\text { prevenção de úlcera por } \\
\text { pressão sacral }\end{array}$ & $\begin{array}{c}\text { INOUE; } \\
\text { MATSUDA, } 2016\end{array}$ & SCIELO & $\begin{array}{l}\text { As coberturas mais caras são as de filme, que geram um } \\
\text { custo de 190,40 reais por paciente. Sendo assim, conclui-se } \\
\text { que as placas de hidrocoloide constituem uma alternativa } \\
\text { mais barata de prevenção da LPP que também apresenta } \\
\text { eficácia, em contrapartida, há necessidade de avaliação } \\
\text { constante da integridade da cobertura por conta do } \\
\text { descolamento, outra medida de prevenção é reduzir a } \\
\text { umidade da pele para a placa ter maior adesão. }\end{array}$ \\
\hline 4 & $\begin{array}{l}\text { Pressure ulcer in } \\
\text { Intensive Care Units: a } \\
\text { case-control study }\end{array}$ & PACHÁ et al., 2018 & SCIELO & $\begin{array}{l}\text { De todas as variáveis analisadas, a maior correlação foi } \\
\text { observada entre a idade e tempo de internação. Quanto maior } \\
\text { ambas as variáveis, maior o risco de desenvolvimento de } \\
\text { lesão por pressão. Além disso quando o motivo de } \\
\text { internação foi relacionado as infecções e neoplasias } \\
\text { aumentam esse risco. }\end{array}$ \\
\hline
\end{tabular}




\begin{tabular}{|c|c|c|c|c|}
\hline & TÍTULO & AUTOR/ ANO & $\begin{array}{c}\text { BASE DE } \\
\text { DADOS }\end{array}$ & RESULTADOS \\
\hline 5 & $\begin{array}{l}\text { A visão dos enfermeiros } \\
\text { quanto a aplicação da } \\
\text { escala de Braden no } \\
\text { paciente idoso }\end{array}$ & DEBON et al., 2018 & LILACS & $\begin{array}{l}\text { Os } 14 \text { enfermeiros entrevistados relataram que } \\
\text { compreendem a relevância da aplicação da escala de Braden, } \\
\text { principalmente nos idosos com internação prolongada, } \\
\text { entretanto, nem todos realizam a aplicação dela. Dos } \\
\text { profissionais que documentam o resultado da escala, eles } \\
\text { alegam falta de tempo para realizar as intervenções para cada } \\
\text { score, ou seja, o cuidado não é aplicado. }\end{array}$ \\
\hline 6 & $\begin{array}{l}\text { Nursing diagnosis in } \\
\text { older adults risk for } \\
\text { pressure injury }\end{array}$ & GARCIA et al., 2021 & SCIELO & $\begin{array}{c}\text { O maior risco de desenvolvimento da LPP foi atribuído a } \\
\text { mulheres idosas, acima de } 80 \text { anos, estas apresentavam com } \\
\text { maior frequência o risco moderado segundo a escala de } \\
\text { Braden. Das atribuições do enfermeiro, foram destacadas as } \\
\text { que estimulam a movimentação e aumento da circulação, } \\
\text { hábito de higiene satisfatórios, controle da pressão, } \\
\text { hidratação, entre outros. }\end{array}$ \\
\hline 7 & $\begin{array}{l}\text { Úlcera por pressão em } \\
\text { unidade de terapia } \\
\text { intensiva: estudo } \\
\text { epidemiológico }\end{array}$ & PETZ et al., 2017 & BDENF & $\begin{array}{l}\text { Dos sete participantes que desenvolveram LPP, } 71,4 \% \text { eram } \\
\text { pessoas idosas, o tempo médio de internação desses } \\
\text { pacientes foi de } 15,2 \text { dias. A região mais afetada foi a sacral } \\
\text { identificando a importância de realizar a mudança de } \\
\text { decúbito para prevenir o aumento da pressão enquanto o } \\
\text { paciente estiver acamado. }\end{array}$ \\
\hline 8 & $\begin{array}{l}\text { Knowledge of the } \\
\text { nursing team on pressure } \\
\text { ulcer prevention }\end{array}$ & $\begin{array}{l}\text { GALVÃO et al., } \\
2017\end{array}$ & SCIELO & $\begin{array}{c}\text { Dos } 40 \text { profissionais, sendo } 14 \text { enfermeiros e } 26 \\
\text { técnicos/auxiliares de enfermagem, apenas } 51,4 \% \\
\text { enfermeiros e } 63,4 \% \text {. técnicos/auxiliares sabiam as medidas } \\
\text { de profilaxia para LPP. O baixo conhecimento da prevenção } \\
\text { contribui para o surgimento e agravo das LPP } \\
\text { principalmente nos pacientes em internação prolongada. }\end{array}$ \\
\hline 9 & $\begin{array}{l}\text { Ocorrência de lesão por } \\
\text { pressão em unidade de } \\
\text { terapia intensiva de um } \\
\text { hospital universitário }\end{array}$ & FARIAS et al., 2017 & LILACS & $\begin{array}{l}\text { Identificou-se maior frequência de LPP em mulheres idosas, } \\
\text { com comorbidades. A região mais afetada dos pacientes foi a } \\
\text { sacro }(62,5 \%) \text { seguido dos calcâneos }(20,83 \%) \text { e o estágio } \\
\text { mais aparente foi o II ( } 45,83 \%) \text {. Quanto ao tempo de } \\
\text { internação a média foi de } 17,4 \text { dias e } 10 \text { pacientes } \\
\text { desenvolveram as lesões durante internação na UTI. }\end{array}$ \\
\hline 10 & $\begin{array}{l}\text { Ocorrência de } \\
\text { lesão por pressão em } \\
\text { pacientes internados em } \\
\text { unidade de terapia } \\
\text { intensiva }\end{array}$ & SANTOS et al., 2021 & SCIELO & $\begin{array}{l}\text { O perfil epidemiológico mais frequente foi definido nas } \\
\text { mulheres idosas, acima de } 65 \text { anos com comorbidades pré- } \\
\text { existentes e a região mais afetada foi a sacral. Os } \\
\text { pesquisadores concluíram que cada dia a mais de internação } \\
\text { aumentava o risco em } 3,5 \text { vezes para o surgimento da lesão, } \\
\text { por isso a medida de prevenção mais citada no estudo foi } \\
\text { trabalhar com a equipe para reduzir o tempo de internação }\end{array}$ \\
\hline
\end{tabular}

Fonte: Sampaio et al. (2021).

\section{Discussão}

Foi possível observar após a coleta de dados que os artigos possuíam duas temáticas centrais, descrever as contribuições do enfermeiro frente a prevenção de LPP nos pacientes idosos internados na UTI e os cuidados de enfermagem comumente adotados. Como outras abordagens interessantes, está descrito na literatura o perfil epidemiológico desses pacientes, fatores de 
risco para LPP e as causas multifatoriais que interferem a sistematização do cuidar do profissional enfermeiro. Sendo assim, a discussão a seguir será apresentada em subtópicos, cada um segundo os artigos encontrados na literatura e inclusos nesta revisão.

\subsection{Cuidados de Enfermagem e Profilaxia}

A enfermagem se faz presente dentro da equipe multidisciplinar com foco no atendimento humanizado ao paciente hospitalizado com risco ou que possua a lesão já instalada e em tratamento, dessa maneira protocolos a comitiva de feridas formada por enfermeiros estomaterapeutas permite avaliação mais rigorosa com diminuição dos riscos e permitindo assim a prevenção desses tipos de lesões. (Corrêa et al. 2018).

Um dos métodos mais eficazes para prevenção da LPP é a educação em saúde, o enfermeiro é um profissional habilitado para informar e orientar os acompanhantes e familiares acerca dos riscos da não adesão as orientações de saúde prescritas pelos profissionais (Nettina, 2015).

Nesta revisão, o estudo de Garcia et al. (2021) descreve as principais medidas profiláticas como o monitoramento da integridade da pele através da escala de Braden, estímulo a exercícios físicos que melhorem a função circulatória do corpo, aumento da hidratação da pele, regulação da temperatura, controle da pressão exercida sobre o tecido e manutenção da higiene satisfatória.

Além do monitoramento e controle da pressão do paciente sobre o leito a mudança de decúbito é descrita na literatura como método eficaz e de responsabilidade da equipe de enfermagem. No estudo de Sanches et al. (2018) os profissionais aderiram ao protocolo de alívio da pressão de forma correta a cada duas horas, para isso a instituição investiu em um relógio próprio para cada paciente, esta medida teve uma elevada taxa de adesão e como resultado apenas 5,22\% dos pacientes desenvolveram LPP durante a internação. evidenciando a importância do conhecimento científico e aplicação desses conhecimentos na prática, o que resulta na maior qualidade da internação dos pacientes.

Esses trabalhos corroboram com o de Petz et al. (2018) que identificou que a região sacral foi a mais afetada dentre os pacientes estudados, esta área localizada próxima a uma proeminência óssea acaba sofrendo com o atrito e pressão, além do aumento da temperatura, portanto, para reduzir esse dano, a melhor medida adotada é a mudança de decúbito.

Farias et al. (2017) concluiu que não somente a região sacral, mas os calcâneos também são áreas comumente afetadas por lesões, destacando que $52,94 \%$ destes pacientes vieram a óbito. Farias e colaboradores descrevem que a falta de conhecimento e destreza para aplicar os cuidados de enfermagem é uma das causas desse desfecho clínico.

Nesta revisão o estudo Galvão et al. (2017) complementa o de Farias através da avaliação do conhecimento de profissionais da enfermagem sobre as medidas preventivas da LPP, Galvão concluiu que apenas $51,4 \%$ dos enfermeiros participantes responderam os questionamentos corretamente, além disso estes dados ratificam o apresentado por Debon et al. (2018) que diz que a assistência de enfermagem precisa ser atualizada e específica para cada paciente e apresentar destreza para executar as atividades do plano de cuidados.

Quando não é possível intervir o surgimento da lesão através de medidas preventivas, cabe ao profissional identificar a lesão preferencialmente em estágios iniciais, e consequentemente elaborar um plano de cuidados afim de obter a reeptelização tecidual em menor tempo sem negligenciar a ferida independentemente do seu estágio (Feitosa et al., 20020).

Neste sentido, a escala de Braden contribui significativamente para o monitoramento dessas lesões e condutas de enfermagem, entretanto, neste estudo foi encontrada uma referência que descreve o cenário de saúde atual, descrito por Debon et al. (2018) que avaliou a adesão da escala no processo de enfermagem e relatou que os profissionais reconhecem a importância da avaliação tissular, principalmente nos idosos em UTI, mas alegam falta de tempo para documentação e prática das atividades propostas após a pontuação do score. Sem a evolução diária do paciente, não há registro de como a pele estava no momento da admissão, isto dificulta a identificação de sinais flogísticos e favorece a progressão da ferida. 
Outras medidas profiláticas encontradas a partir desta busca bibliográfica, foi a aplicação preventiva de coberturas a base de filme e hidrocoloide. A avaliação do custo médio mostrou que em um setor foi investido 2.252 reais, onde o gasto por paciente foi igual a 190 reais. Compreende-se que é um método eficaz e que nestes casos o controle da umidade da pele é fundamental para aumentar a durabilidade das placas, porém este método pode apresentar baixa acessibilidade, principalmente em serviços públicos de saúde, portanto, o profissional deve complementar essas medidas baseando-se no que está disponível para o uso rotineiro e elaborar cuidados que exijam menos intervenção financeira (Inque; Matsuda, 2016).

\subsection{Identificação dos Fatores de Risco para (Lpp) como Medida Profilática}

A pressão exercida sobre o tecido pode afetar a sua integridade, entretanto outros fatores extrínsecos também são responsáveis pelo aparecimento de lesões cutâneas. Grden et al. (2020) identificou que 10,5\% dos casos de LPP em uma unidade de terapia intensiva tinham como causa principal a fricção da pele sobre o leito ou dispositivos médicos, outro achado importante foi a relação do tempo de internação na UTI com a presença de lesões cutâneas, onde entre 1 e 7 dias de internação já foi possível observar essa complicação, isso os autores justificam como baixa massa corpórea dos idosos.

Apesar deste ser o único trabalho que descreve a fricção como fator central do surgimento da LPP, vários outros autores avaliaram o risco da internação prolongada. O tempo de internação foi descrito pelos pesquisadores como um fator de risco altamente ligado as lesões, Pachá et al. (2018) concluiu que quanto maior a idade e tempo de internação, maior risco para LPP, de forma similar Petz et al. (2017) observou que de todos os pacientes avaliados em seu estudo, as lesões cutâneas surgiam com um tempo médio de 15 dias e no trabalho de Farias et al. (2017) a internação prolongada relacionada a LPP foi de 17 dias.

Todos os trabalhos mencionados acima corroboram que o tempo prolongado em uma unidade hospitalar mais especificamente na UTI, onde os pacientes utilizam mais dispositivos e possuem mobilidade reduzida, o risco para o desenvolvimento de iatrogenias é alto, principalmente a perda da integridade cutânea. Vale a pena ressaltar que desses achados, outro ponto em comum está relacionado com as populações estudadas, os pesquisadores concluíam que os mais propensos a ter LPP são as pessoas idosas.

No estudo realizado Santos et al. (2021), foi feita uma análise de risco através de programas estatísticos, eles identificaram que na população idosa, cada dia a mais de internação representava um risco a integridade tissular 3,5 vezes maior, ratificando com os autores desta revisão.

A identificação precoce de um risco a saúde é de suma importância, tendo em vista que direciona a assistência para a prevenção de um agravo. Como a LPP é descrita como uma complicação evitável, a sua profilaxia também inclui caracterizar o perfil epidemiológico desses pacientes afim de elaborar um plano de cuidado específico de acordo com as características de cada paciente.

Quando comparado a idade, Sanches et al. (2018) na análise do perfil epidemiológico dos pacientes com LPP, mostrou que o grupo com faixa etária 41-60 anos foi mais afetado quando comparado aos pertencentes da faixa entre 61-80 anos, estes dados corroboram com Pachá et al. (2018) que também identificou esse risco em idosos acima de 60 anos internados em UTI, Petz et al. (2017) e Garcia et al. (2021), que concluíram que o grupo mais propenso possuía idade acima de 70 e 80 anos respectivamente. Com isso é possível afirmar que quanto maior a idade, maior o risco devido o processo de envelhecimento tegumentar.

Quanto ao sexo mais frequente, Pachá et al. (2018) e Sanches et al. (2018) descrevem mais da metade dos casos de LPP relacionado ao sexo masculino, esses dados diferem dos apresentados por Santos et al. (2021) e Farias et al. (2017) que demonstraram o sexo feminino como o mais acometido. Essas divergências se dão pelo fato do $\mathrm{n}$ amostral ser diferente, em unidades diversas, motivo de internação distinto e condições de saúde individualizadas, entretanto, não foi possível constatar pré-disposição para nenhum dos sexos. 
Quando comparado as comorbidades dos idosos estudados, Santos et al. (2021) observou que os pacientes que possuem lesão renal, diabetes, vítimas de infarto e AVE apresentam maior risco de perder a integridade da pele, de forma complementar, Pachá et al. (2018) considerou o motivo de internação e mostrou que pessoas hospitalizadas por motivos de infecções e neoplasias também apresentavam maior pré-disposição de desenvolvimento da LPP.

O diagnóstico médico dos pacientes que dão entrada na UTI já contribui como fator de risco para LPP, devido as medicações envolvidas neste processo, como anticoagulantes, anti-hipertensivos, antiinflamatórios entre outros que retardam o processo de cicatrização, por isso, nestes casos prevenir a lesão é mais importante do que tratar, considerando que não haverá uma reeptelização da lesão de forma simples (Santos et al., 2021).

Com base nos achados apresentados, pode-se afirmar que a idade, sexo, comorbidades, tempo de internação, uso de medicações que interferem a cicatrização são os fatores de risco para lesão por pressão mais descritos na literatura nos últimos cinco anos e que esses fatores previamente identificados contribuem para a prevenção da LPP.

\section{Considerações Finais}

Neste contexto, as ações de gerenciamento e cuidado do enfermeiro está associada a realização da assistência de forma sistemática. As principais contribuições de enfermagem para a prevenção da LPP em idosos internados na UTI e o seu papel no impedimento da progressão da lesão consistem na mudança de decúbito a cada duas horas para descompressão local, controle da pressão, umidade e temperatura da pele, avaliação constante da integridade da pele através da aplicação da escala de Braden e cuidados gerais com a higiene. Portanto, conclui-se que o enfermeiro é um profissional fundamental e habilitado para promover, manter e recuperar a saúde da pele dos indivíduos submetidos a internação prolongada. Diante disso, espera-se que a seguinte pesquisa possa servi de subsídio para nortear futuras pesquisas sobre essa temática, assim como na diminuição da alta incidência de LPP na classe abordada no presente artigo.

\section{Referências}

Andrade, O. G., Marcon, S. S., \& da Silva, D. M. P. (1997). Como os enfermeiros avaliam o cuidado/cuidador familiar. Revista Gaúcha de Enfermagem, 18(2). Burgeson, RE e Christiano, AM (1997). A junção derme-epidérmica. Opinião atual em biologia celular, 9 (5), $651-658$.

Cesarino, C. B., Rodrigues, M. A. S., Mendonça, R. C. H. R., Corrêa, L. C., \& Amorim, R. C. (2005). Percepções dos pacientes em relação à Unidade Terapia Intensiva. Arq Ciênc Saúde, 12(3), 150-3.

Colombrini, M. R. C., Lopes, M. H. B. D. M., \& Figueiredo, R. M. D. (2006). Adesão à terapia antiretroviral para HIV/AIDS. Revista da Escola de Enfermagem da USP, 40, 576-581.

Corrêa, R. U. et al. (2016). A importância da prescrição de enfermagem para um cuidar sistematizado. ANAIS CONGREGA MIC-ISBN: 978-65-86471-05-2 e ANAIS MIC JR.-ISBN: 978-65-86471-06-9, 1.

Cruz, N. M., Cruz, J. D. A., Carmo, A. D. F. S., Moura, L. A., de Alencar Lubarino, D., \& Brito, R. K. M. (2015). Prevenção de úlceras por pressão e segurança do paciente: percepções de enfermeiros em terapia intensiva. Revista Contexto \& Saúde, 15(28), 62-66.

Debon, R., Fortes, VLF, Rós, ACR, \& Scaratti, M. (2018). A visão de enfermeiros quanto a uma aplicação da escala de Braden no paciente idoso. Revista de Pesquisa: Cuidado é Fundamental, 817-823.

Farias, A. D. A. D., Leal, N. T. B., Travassos, N. P. R., Farias, A. J. A. D., Nobre, A. M. D., \& Almeida, T. D. C. F. (2019). Ocorrência de lesões por pressão em unidade de terapia intensiva de um hospital universitário. Nursing (Säo Paulo), 2927-2931.

Favreto, F. J. L., Betiolli, S. E., Silva, F. B., \& Campa, A. (2017). O papel do enfermeiro na prevenção, avaliação e tratamento das lesões por pressão. RGS, 17(2), 37-47.

Feitosa, D. V., de Oliveira Silva, N. S., Pereira, F. N. M., Almeida, T. F., \& dos Santos Estevam, A. (2020). Atuação do enfermeiro na prevenção de lesão por pressão: uma revisão integrativa da literatura. Revista Eletrônica Acervo Saúde, (43), e2553-e2553.

Feitosa, L. (2015). Humanização Nos Hospitais. Ceará-Fortaleza: livro técnico.

Fernandes, L. M., da Silva, L., de Oliveira, J. L. C., de Souza, V. S., \& Nicola, A. L. (2016). Associação entre predição para lesão por pressão e marcadores bioquímicos. Rev Rene 2 17(4), 490-497. 
Fontela, PC, Abdala, FANB, Forgiarini, SGI, \& Forgiarini, LA (2018). Qualidade de vida em sobreviventes após um período de internação em unidade de terapia intensiva: uma revisão sistemática. Revista Brasileira de terapia intensiva, 30 , 496-507.

Furuya, R. K., Birolim, M. M., Biazin, D. T., \& Rossi, L. A. (2011). A integralidade e suas interfaces no cuidado ao idoso em unidade de terapia intensiva. Rev. enferm. UERJ, 157-161.

Galvão, C. M., Sawada, N. O., \& Trevizan, M. A. (2004). Revisão sistemática: recurso que proporciona a incorporação das evidências na prática da enfermagem. Revista Latino-americana de enfermagem, 12, 549-556.

Galvão, NS, Serique, MAB, Santos, VLCDG, \& Nogueira, PC (2017). Conhecimento da equipe de enfermagem sobre prevenção de úlcera por pressão. Revista Brasileira de enfermagem, 70, 294-300.

Garcia, EDQ, Tarouco da Silva, B., Gautério Abreu, DP, Roque, TDS, dos Santos Sousa, JI, \& Ilha, S. (2021). Diagnóstico de enfermagem em idosos com risco de lesão por pressão. Revista da Escola de Enfermagem da USP, 55.

Gomes, F. S. L., Bastos, M. A. R., Matozinhos, F. P., Temponi, H. R., \& Velásquez-Meléndez, G. (2011). Avaliação de risco para úlcera por pressão em pacientes críticos. Revista da Escola de Enfermagem da USP, 45, 313-318.

Grden, C. R. B., Rodrigues, C. R. B., Ivastcheschen, T., Cabral, L. P. A., Bordin, D., \& Reche, P. M. (2020). Lesões por fricção em idosos internados: estudo transversal. Online braz. j. nurs.(Online).

Inoue, K. C., \& Matsuda, L. M. (2016). Custos de coberturas para a prevenção de úlcera por pressão sacral. Revista Brasileira de Enfermagem, 69 , 641-645. Lima, T. J. V. D., Arcieri, R. M., Garbin, C. A. S., \& Moimaz, S. A. S. (2010). Humanização na atenção à saúde do idoso. Saúde e Sociedade, 19(4), 866-877.

Luiz, FF, Caregnato, RCA, \& Costa, MRD (2017). Humanização na Terapia Intensiva: percepção do familiar e do profissional de saúde. Revista Brasileira de Enfermagem, 70, 1040-1047.

Machado, E. R., \& Soares, N. V. (2016). Humanização em UTI: sentidos e significados sob a ótica da equipe de saúde. Revista de Enfermagem do Centro-Oeste Mineiro, 6(3).

Mallmann, D. G., Galindo Neto, N. M., Sousa, J. D. C., \& Vasconcelos, E. M. R. D. (2015). Educação em saúde como principal alternativa para promover a saúde do idoso. Ciência \& Saúde Coletiva, 20, 1763-1772.

Medeiros, K. K. A. S., Pinto, E. P., Bousquat, A., \& Medina, M. G. (2017). O desafio da integralidade no cuidado ao idoso, no âmbito da Atenção Primária à Saúde. Saúde em debate, 41, 288-295.

Miranda, GMD, Mendes, ADCG, \& Silva, ALAD (2016). O envelhecimento populacional brasileiro: desafios e consequências atuais e futuras. Revista brasileira de geriatria e gerontologia, 19, 507-519.

Motta, J. O. C., Campbell, G. A. M., Caldas, G., \& Guimarães, R. M. (1995). A pele do idoso: a propósito de 150 observações. An Bras Dermatol.

Pachá, HHP, Faria, JIL, Oliveira, KAD, \& Beccaria, LM (2018). Úlcera por pressão em unidades de terapia intensiva: um estudo caso-controle. Revista brasileira de enfermagem, 71, 3027-3034.

Petz, F. D. F. C., Crozeta, K., Meier, M. J., Lenhani, B. E., Kalinke, L. P., \& Pott, F. S. (2017). Úlcera por pressão em unidade de terapia intensiva: estudo epidemiológico. Rev Enferm UFPE, 11(1), 287-95.

Salicio, D. M. B., \& Gaiva, M. A. M. (2006). O significado de humanização da assistência para enfermeiros que atuam em UTI. Revista Eletrônica de Enfermagem, 8(3).

Santos, S., da Rocha Tonhom, S. F., \& Komatsu, R. S. (2016). Saúde do idoso: reflexões acerca da integralidade do cuidado. Revista Brasileira em Promoção da Saúde, 29, 118-127.

Saurusaitis, A. D., Santiago, L. C., Peregrino, A. A. D. F., Silva, R. C. L. D., \& Schutz, V. (2019). Diarreia: dermatite associada à incontinência e lesão por pressão. Rev. enferm. UFPE on line, 1-6.

Silva, A. S. T. D., França, F. L. D., Sousa, D. L. B. D., Simião, C. K. D. S., Silva, R. D. K. S. D., Davim, R. M. B., \& Silva, R. E. M. N. (2018). Acolhimento ao idoso em unidades de saúde da família. Rev. enferm. UFPE on line, 2247-2256.

Silva, R. C. L. D., Figueiredo, N. M. A., \& Meireles, I. B. (2009). Feridas: fundamentos e atualizações em enfermagem. In Feridas: fundamentos e atualizações em enfermagem (pp. 508-508).

Snaches, B., Contrin, L. M., Beccaria, L. M., Frutuoso, I. S., da Silveira, A. M. R., \& Werneck, A. L. (2018). Adesão da enfermagem ao protocolo de lesão por pressão em unidade de terapia intensiva. Arquivos de Ciências da Saúde, 25(3), 27-31.

Sousa, L. M. M., Marques-Vieira, C. M. A., Severino, S. S. P., \& Antunes, A. V. (2017). A metodologia de revisão integrativa da literatura em enfermagem. $N^{\circ} 21$ Série 2-Novembro 2017, 17.

Teixeira, A. K. S., Nascimento, T. D. S., Sousa, I. D., Sampaio, L. R. L., \& Pinheiro, A. R. M. (2017). Incidência de lesões por pressão em Unidade de Terapia Intensiva em hospital com acreditação. Rev Estima, 15(2), 152-60.

Vieira, P., \& de Almeida, M. A. R. (2020). Humanização da assistência de enfermagem em pacientes idosos. Revista de Iniciação Científica e Extensão, 3(1), 371-8. 